\title{
超临界二氧化碳中羰基钴催化的端基炔烃环三聚反应研究
}

\author{
王亚琦 $尹$ 强郭墩 韩利民 \\ 孙琪 洪海龙索全伶* \\ (内蒙古工业大学化工学院 呼和浩特 010051)
}

\begin{abstract}
摘要 原子经济性良好的炔烃 $[2+2+2]$ 环加成反应与绿色溶剂超临界二氧化碳相结合, 是一个符合绿色化学原则的环 境友好反应过程. 建立了一个纯超临界二氧化碳介质中八羰基二钻催化的端基炔烃环三聚反应体系, 在优化的反应条 件下，以较高产率选择性地制备 1,2,4-三取代苯衍生物。优化了催化剂用量、二氧化碳压力、反应温度及时间等反应条 件, 讨论了反应物料及催化剂在超临界二氧化碳介质中的溶解性和相行为, 提出了端基炔烃环三聚反应机理, 并将反 应底物从 $\mathrm{C} \equiv \mathrm{C}$ 键拓展至 $\mathrm{C} \equiv \mathrm{N}$ 键, 对超临界二氧化碳介质中炔-腈环加成反应进行了初步探索. 优化出的炔烃环三聚 催化反应体系无需使用有机助溶剂和各类助剂, 底物适应性好, 产物选择性高, 为合成 1,2,4-三取代苯衍生物提供了一 种绿色合成方法
\end{abstract}

关键词＼cjkstart超临界二氧化碳; 端基炔烃; 环三聚反应; 嶡基钴催化剂; 炔-腈环加成反应

\section{Carbonyl Cobalt-Catalyzed Cyclotrimerization of Terminal Alkynes in Supercritical Carbon Dioxide}

\author{
Wang, Yaqi \\ Yin, Qiang \\ Guo, Dun \\ Han, Limin \\ Sun, Qi \\ Hong, Hailong \\ Suo, Quanling* \\ (Chemical Engineering College, Inner Mongolia University of Technology, Hohhot 010051)
}

\begin{abstract}
Atom-efficient $[2+2+2]$ cycloaddition reaction of alkynes in green solvent supercritical carbon dioxide $\left(\mathrm{ScCO}_{2}\right)$ is an environmentally friendly reaction process that conforms to the principles of green chemistry. Cyclotrimerization of terminal alkynes catalyzed by $\mathrm{Co}_{2}(\mathrm{CO})_{8}$ in pure $\mathrm{ScCO}_{2}$ has been studied to obtain 1,2,4-trisubstituted benzene derivatives with excellent selectivity. The reaction conditions for the cyclotrimerization were optimized, such as concentration of catalyst, $\mathrm{CO}_{2}$ pressure, reaction temperature and time. The solubility and phase behavior of the reaction materials and catalysts in $\mathrm{ScCO}_{2}$ medium were discussed, and the mechanism of $\mathrm{Co}_{2}(\mathrm{CO})_{8}$ catalyzed cyclotrimerization of terminal alkynes was assumed. The reaction substrate was extended from $\mathrm{C} \equiv \mathrm{C}$ (alkyne) to $\mathrm{C} \equiv \mathrm{N}$ (nitrile), and the alkyne-nitrile cycloaddition reaction in $\mathrm{ScCO}_{2}$ was preliminary explored. Our optimized catalytic system for the cyclotrimerization of terminal alkynes exhibited wide substrate scope and high product selectivity, in which no organic co-solvent or additives were added. It provided a green synthetic method for 1,2,4-trisubstituted benzenes.

Keywords supercritical carbon dioxide; terminal alkyne; cyclotrimerization reaction; carbonyl cobalt catalyst; alkyne-nitrile cycloaddition reaction
\end{abstract}

芳香烃类化合物广泛存在于自然界中，很多植物的 药用功能都与含芳香环或芳香杂环的结构单元有关 ${ }^{[1]}$. 因此，研究制备各类芳香或杂环芳香化合物一直备受化 学家关注. 过渡金属催化的炔烃 $[2+2+2]$ 环加成反应 是合成多取代苯和吡啶衍生物的经典方法，只经一步反
应就将直链炔烃转变成取代芳香化合物 ${ }^{[2]}$. 通过选择不 同取代基的炔烃底物设计 $[2+2+2]$ 环加成反应，在新 构建的芳环上同时引入多个不同取代基，具有极高的原 子经济性. 为了提高 $[2+2+2]$ 环加成反应的产物选择 性，人们研究了含钴、镍、钉、钯、钛等过渡金属的诸

\footnotetext{
* Corresponding author. E-mail: szj@imut.edu.cn

Received April 9, 2019; revised May 14, 2019; published online May 28, 2019.

Project supported by the National Natural Science Foundation of China (No. 21266019) and the Natural Science Foundation of Inner Mongolia Autonomous Region of China (No. 2015MS0204).

国家自然科学基金(No. 21266019)、内蒙古自然科学基金(No. 2015MS0204)资助项目.
} 
多催化剂, 发现了底物适应范围宽、反应转化率和选择 性均较高的催化体系，并使这一合成路线成为制备苯、 吡啶衍生物的通用方法 ${ }^{[3]}$.

随着人们环保意识的不断提高, 绿色化学被大力提 倡并得到了迅速的发展 ${ }^{[4]}$. 在有机化学反应中, 溶剂起 着非常重要的媒介作用, 反应产物的提纯与精制过程也 是在有机溶剂中完成. 医疗药品、农药和精细化学品等 工业生产中大量使用传统有机溶剂是造成我国雾䨍等 大气环境严重污染的主要因素之一. 因此, 使用环境友 好溶剂替代传统有机溶剂已经成为绿色化学发展的重 要方向 ${ }^{[5]}$. 超临界二氧化碳(supercritical carbon dioxide, $\mathrm{ScCO}_{2}$ ) 具有廉价、无毒、不燃、溶解性好、易与产物分 离等特点, 是理想的环境友好溶剂 ${ }^{[6]}$. 以超临界二氧化 碳作为反应介质的研究报道涉及诸多有机合成反应 ${ }^{[7]}$, 如加氢反应 ${ }^{[8]}$ 、氧化反应 ${ }^{[9]}$ 、羰基化反应 ${ }^{[10]}$ 和形成碳碳 键反应 ${ }^{[11]}$ 等, 但有关炔烃 $[2+2+2]$ 环加成反应的研究 相对较少, 仅有 3 个课题组对炔烃环三聚反应进行了报 道 ${ }^{[12]}$.

炔烃环三聚反应通常会产生 $1,3,5$-三取代苯和 1,2,4-三取代苯两种环加成反应产物, 通过选择适宜的 催化反应体系来提高产物选择性一直是化学家们关注 的重点. 江焕峰小组 ${ }^{[2 \mathrm{a}, 12 \mathrm{~b}]}$ 在 $\mathrm{PdCl}_{2}$ 为催化剂, $\mathrm{CuCl}_{2}$ 为助 催化剂, $\mathrm{ScCO}_{2}$ 为反应溶剂, 甲醇为助溶剂的反应条件 下, 实现了炔烃环三聚反应. 在优化的反应条件下, 江 焕峰课题组以 $51 \% \sim 97 \%$ 的产率得到相应的 1,3,5-三取 代苯衍生物. 该催化反应体系需要甲醇作助溶剂, 反应 溶剂不能完全绿色化, 也不能发生苯乙炔的环三聚反 应. 李金恒课题组 ${ }^{[12 c]}$ 研究了以纯 $\mathrm{ScCO}_{2}$ 为反应介质, $\mathrm{PdBr}_{2}$ 为催化剂的炔烃环三聚反应. 研究结果表明, 在 $\mathrm{ScCO}_{2}$ 介质中使用活性更高的 $\mathrm{PdBr}_{2}$ 催化剂, 可实现苯 乙炔、对甲基苯乙炔和 1-庚炔等底物的环三聚反应, 主 产物为 1,3,5-三取代苯衍生物. 该反应体系催化剂用量 较高, 反应时间较长. Avilés 等 ${ }^{[12 \mathrm{~d}]}$ 报道了在 $\mathrm{ScCO}_{2}$ 中选 用二羰基环戊二烯基钴 $\mathrm{CpCo}(\mathrm{CO})_{2}(\mathrm{Cp}=$ 环戊二烯基)为 催化剂的炔烃环三聚反应. 在高温、高压及光助催化的 作用下, 苯乙炔仅以 $22 \%$ 的总产率得到两种异构体的混 合物, 其中 1,2,4-三取代苯和 1,3,5-三取代苯的比例为 $5: 1$. 其它炔烃大多总收率较低(小于 $20 \%$ ). 前人研究 结果表明, 在 $\mathrm{ScCO}_{2}$ 中进行的炔烃环三聚反应, 产物选 择性与催化剂类型有关, 当选用钯盐 $\left(\mathrm{PdCl}_{2} 、 \mathrm{PdBr}_{2}\right)$ 为催 化剂时, 产物选择性地生成 1,3,5-三取代苯; 当选用羰 基钴催化剂 $\mathrm{CpCo}(\mathrm{CO})_{2}$ 时, 可得到 1,2,4-三取代苯为主 产物的异构体混合物, 其中两种异构体 1,2,4-三取代苯 和 1,3,5-三取代苯的比例在 3 5 之间.

将原子经济性良好的炔烃 $[2+2+2]$ 环加成反应与
绿色溶剂超临界二氧化碳结合起来, 是一个符合绿色化 学原则的环境友好反应过程, 这方面的研究引起了我们 课题组的关注. 本文通过对常见钴催化剂的篮选及反应 条件的优化, 发现了一个以纯 $\mathrm{ScCO}_{2}$ 为反应介质, $\mathrm{Co}_{2}(\mathrm{CO})_{8}$ 为催化剂, 可高选择性地制备 $1,2,4$-三取代苯 的新型催化反应体系. 本文优化出的端炔环三聚 $\mathrm{ScCO}_{2}$ 催化反应体系, 具有催化剂廉价易得、无需助溶剂和各 类助剂、产物选择性高等优点. 本文通过高压可视反应 釜，对 $\mathrm{ScCO}_{2}$ 介质中反应物料和催化剂的溶解过程及相 变化进行了观察分析, 提出了炔烃 $[2+2+2]$ 环三聚反 应机理，并将底物范围拓展至炔-腈环加成反应.

\section{1 结果与讨论}

\section{1 超临界二氧化碳中炔烃环三聚反应}

\subsection{1 反应条件优化及溶解性和相行为研究}

本文以纯 $\mathrm{ScCO}_{2}$ 介质中苯乙炔的环三聚反应为模 型，对几种常见的钴催化剂进行篮选，并优化了反应条 件, 结果列于表 1. 超临界二氧化碳介质中物料的溶解 性和化学反应的相行为对于研究催化反应机理十分重

表 1 反应条件的优化 ${ }^{a}$

Table 1 Optimization of reaction conditions

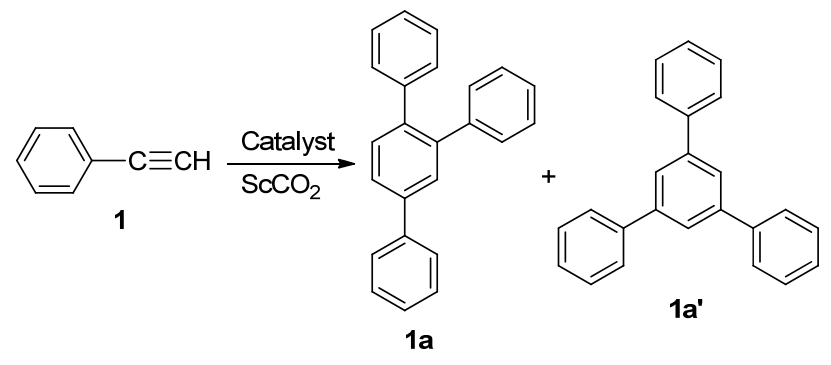

\begin{tabular}{clcccc}
\hline Entry & Catalyst $(\mathrm{mol} \%)$ & $P_{\mathrm{CO}_{2}} / \mathrm{MPa}$ & $T /^{\circ} \mathrm{C}$ & $\mathrm{Time} / \mathrm{h}$ & $\begin{array}{c}\mathrm{Yield}^{b} \% \\
\text { of 1a }\end{array}$ \\
\hline 1 & $\mathrm{CoCl}_{2} \cdot 6 \mathrm{H}_{2} \mathrm{O}(15)$ & 8 & 80 & 10 & 16 \\
2 & $\mathrm{Co}(15)$ & 8 & 80 & 10 & 21 \\
3 & $\mathrm{CpCo}(\mathrm{CO})_{2}(15)$ & 8 & 80 & 10 & 47 \\
$\mathbf{4}$ & $\mathrm{Co}_{2}(\mathrm{CO})_{8}(\mathbf{1 5})$ & $\mathbf{8}$ & $\mathbf{8 0}$ & $\mathbf{1 0}$ & $\mathbf{6 2}$ \\
5 & $\mathrm{Co}_{2}(\mathrm{CO})_{8}(5)$ & 8 & 80 & 10 & 15 \\
6 & $\mathrm{Co}_{2}(\mathrm{CO})_{8}(10)$ & 8 & 80 & 10 & 25 \\
7 & $\mathrm{Co}_{2}(\mathrm{CO})_{8}(20)$ & 8 & 80 & 10 & 52 \\
8 & $\mathrm{Co}_{2}(\mathrm{CO})_{8}(15)$ & 8 & 60 & 10 & 19 \\
9 & $\mathrm{Co}_{2}(\mathrm{CO})_{8}(15)$ & 8 & 70 & 10 & 43 \\
10 & $\mathrm{Co}_{2}(\mathrm{CO})_{8}(15)$ & 8 & 90 & 10 & 59 \\
11 & $\mathrm{Co}_{2}(\mathrm{CO})_{8}(15)$ & 10 & 80 & 10 & 45 \\
12 & $\mathrm{Co}_{2}(\mathrm{CO})_{8}(15)$ & 12 & 80 & 10 & 16 \\
13 & $\mathrm{Co}_{2}(\mathrm{CO})_{8}(15)$ & 8 & 80 & 6 & 38 \\
14 & $\mathrm{Co}_{2}(\mathrm{CO})_{8}(15)$ & 8 & 80 & 8 & 42 \\
15 & $\mathrm{Co}_{2}(\mathrm{CO})_{8}(15)$ & 8 & 80 & 12 & 46 \\
\hline
\end{tabular}

${ }^{a}$ Reaction conditions: $\mathrm{PhC} \equiv \mathrm{CH}\left(\mathrm{Ph}=\right.$ phenyl) $1.8 \mathrm{mmol} ;{ }^{b}$ Isolated yield, 1a' was detected by TLC and GC-MS technology. 
要 $^{[13]}$, 我们利用高压可视反应釜, 对反应物、产物和催 化剂的溶解性及反应过程的相变化进行了观察分析.

在纯 $\mathrm{ScCO}_{2}$ 介质中, 我们选择了四种不同的钴催化 剂: $\mathrm{CoCl}_{2} \cdot 6 \mathrm{H}_{2} \mathrm{O}$ 、钴粉、 $\mathrm{CpCo}(\mathrm{CO})_{2}$ 和 $\mathrm{Co}_{2}(\mathrm{CO})_{8}$ 进行苯 乙炔环三聚催化反应研究. 实验结果表明, 在 $\mathrm{CO}_{2}$ 压力 为 $8 \mathrm{MPa}$, 催化剂用量为 $15 \mathrm{~mol} \%$, 反应温度 $80{ }^{\circ} \mathrm{C}$, 反 应时间 $10 \mathrm{~h}$ 的条件下, 四种催化剂均能使苯乙炔发生环 三聚反应, 产物以 1,2,4-三苯基苯(1a)为主, 另一种异构 体 1,3,5-三苯基苯 $\left(\mathbf{1 a}^{\prime}\right)$ 很少, 只能通过 GC-MS 检测到. 无机盐 $\mathrm{CoCl}_{2} \cdot 6 \mathrm{H}_{2} \mathrm{O}$ 或钴粉作催化剂时, $1 \mathbf{a}$ 产率不高(表 1 , Entries 1, 2); 羊基钴化合物 $\mathrm{CpCo}(\mathrm{CO})_{2}$ 和 $\mathrm{Co}_{2}(\mathrm{CO})_{8}$ 作催化剂时, $1 \mathrm{a}$ 产率明显提高, 其中 $\mathrm{Co}_{2}(\mathrm{CO})_{8}$ 催化剂以 最高 62\%的产率得到 $\mathbf{1 a}$ (表 1, Entries 3, 4).

利用高压可视釜, 对模型反应中反应物、产物和催 化剂在 $\mathrm{ScCO}_{2}$ 中的溶解性及相变情况进行了观察. 结果 显示, 在反应条件下, 反应物苯乙炔完全溶于 $\mathrm{ScCO}_{2}$ 中 形成均一的单相体系, 产物 $\mathbf{1 a}$ 不溶于 $\mathrm{ScCO}_{2}$, 无机盐催 化剂 $\mathrm{CoCl}_{2} \cdot 6 \mathrm{H}_{2} \mathrm{O}$ 和钴粉在 $\mathrm{ScCO}_{2}$ 中几乎不溶, 羰基钴 催化剂 $\mathrm{CpCo}(\mathrm{CO})_{2}$ 和 $\mathrm{Co}_{2}(\mathrm{CO})_{8}$ 都能很好地溶解于 $\mathrm{ScCO}_{2}$, 并形成均匀的单相体系. 实验结果表明, 无机 盐 $\mathrm{CoCl}_{2} \cdot 6 \mathrm{H}_{2} \mathrm{O}$ 或钴粉作催化剂时, $1 \mathbf{a}$ 产率不高, 可能因 为无机盐或钴粉在 $\mathrm{ScCO}_{2}$ 中溶解度较低, 不能与底物苯 乙炔充分有效接触、碰撞, 导致反应产率较低. 当选用 在 $\mathrm{ScCO}_{2}$ 中溶解性较好的羰基钴 $\mathrm{CpCo}(\mathrm{CO})_{2}$ 或 $\mathrm{Co}_{2}(\mathrm{CO})_{8}$ 催化时, $1 \mathrm{a}$ 产率明显提高. 基于以上研究结果, 本文选择较为廉价易得的 $\mathrm{Co}_{2}(\mathrm{CO})_{8}$ 作为 $\mathrm{ScCO}_{2}$ 中端炔 环三聚反应的催化剂.

采用单因素实验对反应条件进行了优化. 当催化剂 用量为 $15 \mathrm{~mol} \%$ 时, 产物 1a 的产率最高, 达 $62 \%$, 增加 或者减少催化剂用量会导致 1a 产率降低(表 1, Entries $4 \sim 7)$. 反应温度考察结果显示, 当反应温度为 $80{ }^{\circ} \mathrm{C}$ 时, 1a 产率最高(表 1, Entries 5,8 10). 将二氧化碳压力从 $8 \mathrm{MPa}$ 升高至 $12 \mathrm{MPa}, 1 \mathbf{1 a}$ 产率大幅降低(表 1, Entries 4, $11,12)$. 相行为实验显示, 在 $\mathrm{ScCO}_{2}$ 中, 随着反应温度 的升高, 催化剂 $\mathrm{Co}_{2}(\mathrm{CO})_{8}$ 的溶解度逐渐增大, 到 $80{ }^{\circ} \mathrm{C}$ 时则完全溶解, 体系呈现出均匀的深红色, 继续升温, 体系并无明显变化. 但是, 催化剂 $\mathrm{Co}_{2}(\mathrm{CO})_{8}$ 的溶解度受 压力影响较大. 当 $\mathrm{CO}_{2}$ 充入 $8 \mathrm{MPa}$ 时, 体系呈现均匀的 红色, $\mathrm{CO}_{2}$ 压力继续升高, 催化剂 $\mathrm{Co}_{2}(\mathrm{CO})_{8}$ 则析出, 在 反应釜视窗上, 可观察到橙红色 $\mathrm{Co}_{2}(\mathrm{CO})_{8}$ 颗粒, 同时体 系颜色也明显变浅, 这可能因为压力增加, $\mathrm{ScCO}_{2}$ 密度 增大, 导致 $\mathrm{Co}_{2}(\mathrm{CO})_{8}$ 溶解度降低, 从而使反应产率降 低. 当反应时间从 $6 \mathrm{~h}$ 增加到 $10 \mathrm{~h}$ 时, $\mathbf{1 a}$ 的产率逐渐增 大, 继续增加反应时间, 1a 产率反而降低(表 1 , Entries 4, $13 \sim 15)$, 这可能是由于产物 $1 \mathrm{a}$ 与催化剂 $\mathrm{Co}_{2}(\mathrm{CO})_{8}$ 发生
了配位副反应所致 ${ }^{[14]}$. 综合上述实验结果, 本文优化出 的适宜反应条件为: 催化剂 $\mathrm{Co}_{2}(\mathrm{CO})_{8}$ 用量 $15 \mathrm{~mol} \%$ 、 $\mathrm{CO}_{2}$ 压力 $8 \mathrm{MPa}$ 、反应温度 $80{ }^{\circ} \mathrm{C}$ 、反应时间 $10 \mathrm{~h}$.

我们也通过可视反应釜对优化条件下的反应过程 进行了观察: 将底物苯乙炔和催化剂 $\mathrm{Co}_{2}(\mathrm{CO})_{8}$ 放入反 应釜中, 用 $\mathrm{Ar}$ 换气, 然后将反应釜加热至 $50{ }^{\circ} \mathrm{C}$, 再向 釜中缓缓通入二氧化碳至指定压力. 结果显示, 随着 $\mathrm{CO}_{2}$ 压力增加, 底物苯乙炔和催化剂 $\mathrm{Co}_{2}(\mathrm{CO})_{8}$ 逐渐溶 解. 继续升高反应温度至 $80{ }^{\circ} \mathrm{C}$, 体系颜色逐渐由橙红 色变为深红色. 当达到优化反应条件后, 体系呈现出深 红色的均一透明状态. 随着苯乙炔环三聚反应的进行, 产物不断生成, 因环三聚产物不溶于 $\mathrm{ScCO}_{2}$, 可观察到 反应釜视窗逐渐被不透光的颗粒物覆盖. 反应结束, 缓 慢释放 $\mathrm{CO}_{2}$ 后，可在反应釜中看到大量白色针状晶体产 物. 由此可见, 在超临界二氧化碳中, 八羰基二钴的良 好溶解性为其充分发挥催化作用提供了有利条件; 由于 产物不溶于 $\mathrm{ScCO}_{2}$, 反应结束后, 产物直接从反应体系 中析出, 有利于后续的分离与纯化.

\subsection{2 底物适用范围考察}

在优化反应条件下, 我们对底物适用范围进行了考 察, 结果列于表 2. 一系列对位取代苯乙炔也能发生环 三聚反应, 产物均以 1,2,4-三取代苯为主(表 2, Entries 2 5). 苯乙炔对位取代基的电子效应对反应产率有一 定影响, 当苯环对位引入给电子取代基 $\left(\mathrm{CH}_{3}, \mathrm{OCH}_{3}\right)$ 时, 反应产率略有降低(2a: 52\%, 3a: 58\%); 当取代基为拉电 子基( $\mathrm{Br}, \mathrm{F})$ 时，反应产率明显降低(4a: 40\%, 5a: 34\%); 若延长反应时间到 $12 \mathrm{~h}$, 由于 4 -氟苯基乙炔在 $\mathrm{ScCO}_{2}$ 中 有较大的溶解度, 经充分反应后, 4-氟苯基乙炔环三聚 反应的产率从 34\%增加到 67\%(表 2, Entry 5). 杂环芳香 端炔、茂金属端炔和长链脂肪端炔作底物时, 也能以中 等产率得到相应的 1,2,4-三取代苯, 但未检测到异构体 1,3,5-三取代苯(表 2, Entries 6 8). 本文反应体系不仅 适用于液态端炔底物(表 2, Entries 1 3, 5 6, 8), 也同 样适用于固态端炔底物(表 2, Entries 4, 7).

在传统有机溶剂中进行的 $\mathrm{Co}_{2}(\mathrm{CO})_{8}$ 催化不对称炔 烃环三聚反应已有大量报道 ${ }^{[3 \mathrm{a}]}$, 使用的溶剂包括甲苯、 二氯甲烷、1,4-二氧六环等, 环三聚反应产物为 $1,2,4$-和 1,3,5-三取代苯衍生物的混合物, 并以 1,2,4-三取代苯为 主产物. 已报道的有机溶剂中此类反应大多产率中等 $(46 \% \sim 72 \%)$, 但在提高反应温度及延长反应时间的条 件下, 个别底物获得了较高产率 ${ }^{[15]}$. 本文优化出的催化 反应体系与传统有机溶剂体系相比, 炔烃环三聚反应的 产率和选择性相近, 表明 $\mathrm{ScCO}_{2}$ 在催化反应体系中主要 起溶剂的作用. 
表 2 底物适用范围

Table 2 Substrate scope

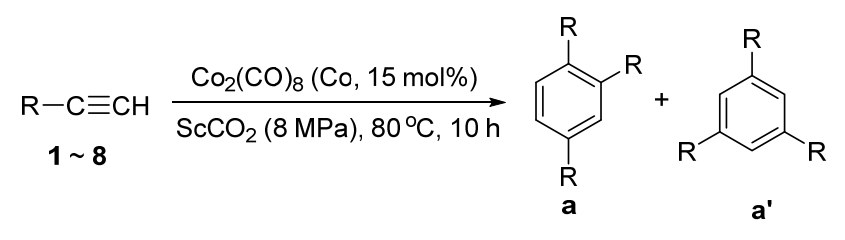

\begin{tabular}{|c|c|c|c|c|}
\hline \multirow{2}{*}{ Entry } & \multirow{2}{*}{ Alkyne } & \multirow{2}{*}{ Product } & \multicolumn{2}{|c|}{ Yield $^{a} / \%$} \\
\hline & & & $\mathbf{a}$ & $\mathbf{a}^{\prime}$ \\
\hline 1 & $\mathrm{HC} \equiv \mathrm{C}-$ & 1a & 62 & Trace \\
\hline 2 & $\mathrm{HC} \equiv \mathrm{C}-$ & $2 a$ & 52 & Trace \\
\hline 3 & $\mathrm{HC} \equiv \mathrm{C}-$ & $3 \mathbf{a}$ & 58 & Trace \\
\hline 4 & $\mathrm{HC} \equiv \mathrm{C}-$ & $4 a$ & 40 & Trace \\
\hline $5^{\mathrm{b}}$ & $\mathrm{HC} \equiv \mathrm{C}-$ & 5a & 67 & Trace \\
\hline 6 & $\mathrm{HC} \equiv \mathrm{C}-$ & $6 a$ & 44 & - \\
\hline 7 & $\mathrm{HC} \equiv \mathrm{C}-$ & $7 \mathbf{a}$ & 53 & - \\
\hline 8 & $\mathrm{HC} \equiv \mathrm{CCH}_{2}\left(\mathrm{CH}_{2}\right)_{3} \mathrm{CH}_{3}$ & $8 \mathbf{a}$ & 57 & - \\
\hline
\end{tabular}

${ }^{a}$ Isolated yield. ${ }^{b}$ Reaction time: $10 \mathrm{~h}$, yield of 5a: $34 \%$; reaction time: $12 \mathrm{~h}$, yield of 5a: $67 \%$.

\subsection{3 底物拓展实验}

炔烃与有机腈进行 $[2+2+2]$ 环加成反应是合成多 取代吡啶的有效方法, 具有极高的原子经济性, 在有机 溶剂为反应媒介的合成中被广泛应用, 但是在绿色溶剂 $\mathrm{ScCO}_{2}$ 中进行的炔-腈环加成反应还未见报道. 我们课 题组曾以甲苯为溶剂, 以 $\mathrm{CpCo}(\mathrm{CO})_{2}$ 或 $\mathrm{Co}_{2}(\mathrm{CO})_{6}\left(\mu_{2}-\right.$ $\left.\eta^{2}-\mathrm{FcC} \equiv \mathrm{CH}\right)(\mathrm{Fc}=$ 二茂铁基)为催化剂, 利用二茂铁基 乙炔 $(\mathrm{FcC} \equiv \mathrm{CH})$ 与有机腈进行 $[2+2+2]$ 环加成反应, 成 功地获得了 4 种多取代吡啶异构体 ${ }^{[16]}$. 在此基础上, 我 们又尝试将环三聚反应底物从 $\mathrm{C} \equiv \mathrm{C}$ 键拓展至 $\mathrm{C} \equiv \mathrm{N}$ 键. 本文以 $\mathrm{ScCO}_{2}$ 作为反应介质, 羰基钴为催化剂, 对炔腈 $[2+2+2]$ 环加成反应进行了研究, 结果见表 3 .

从表 3 可以看出, 将苯乙炔环三聚反应优化出的反 应条件应用于苯乙炔与苯甲腈的环加成反应时, 没有分 离出取代吡啶产物, 只得到了苯乙炔的环三聚反应产物 $\mathbf{1 a}$ (表 3, Entry 1). 将炔烃底物换为二茂铁基乙炔时, 仍 然是只有炔烃环三聚反应产物 7a (表 3, Entry 2). 与没
有苯甲腈底物存在的情况相比较(表 2, Entries 1,7), 1a 和 7a 的产率都明显降低, 这说明炔烃环三聚反应优化 出的催化剂 $\mathrm{Co}_{2}(\mathrm{CO})_{8}$ 并不适合炔-腈环加成反应. 于是 选择了另外两种羰基钴催化剂 $\mathrm{CpCo}(\mathrm{CO})_{2}$ 和 $\mathrm{Co}_{2}(\mathrm{CO})_{6}$ $\left(\mu_{2}-\eta^{2}-\mathrm{FcC} \equiv \mathrm{CH}\right)$, 在 $\mathrm{ScCO}_{2}$ 介质中考察底物 $\mathrm{FcC} \equiv \mathrm{CH}$ 与 $\mathrm{C}_{6} \mathrm{H}_{5} \mathrm{C} \equiv \mathrm{N}$ 进行的环加成反应, 是否能得到取代吡啶 产物. 当 $\mathrm{CO}_{2}$ 压力为 $10 \mathrm{MPa}$, 反应温度为 $110{ }^{\circ} \mathrm{C}$, 摚拌 反应 $10 \mathrm{~h}$ 后, 成功地获得了两种取代吡啶衍生物 2-苯 基-4,6-二二茂铁基吡啶(b)和 2-苯基-3,6-二二茂铁基吡 啶( $\left.\mathbf{b}^{\prime}\right)$, 同时也分离出取代苯衍生物 1,2,4-三二茂铁基苯 (7a)(表 3, Entries 3 4). 在炔-腈环加成反应所获得的两 种吡啶衍生物异构体中, 由于 2,4,6-取代吡啶 (b) 比 2,3,6-取代吡啶 $\left(\mathbf{b}^{\prime}\right)$ 具有更小的空间位阻, 因而产率相对 较高. 将有机溶剂甲苯与绿色溶剂 $\mathrm{ScCO}_{2}$ 中得到的炔腈环加成反应结果进行了对比(表 3 , Entries $5 \sim 7$ ) ${ }^{[16]}$, 可 以看出, 反应的化学选择性主要取决于催化剂的种类和 活性，而溶剂仅对反应主产物的产率影响较大.

在二茂铁基乙炔与苯甲腈的 $[2+2+2]$ 环加成反应 中, 三种羰基钴催化剂表现出了不同的化学选择性. 无 论在 $\mathrm{ScCO}_{2}$ 还是在甲苯溶剂中, $\mathrm{CpCo}(\mathrm{CO})_{2}$ 都对炔-腈环 加成反应表现出较高的催化活性, 产物以吡啶衍生物为 主、苯衍生物为副; 当选用 $\mathrm{Co}_{2}(\mathrm{CO})_{6}\left(\mu_{2}-\eta^{2}-\mathrm{FcC} \equiv \mathrm{CH}\right)$ 作为催化剂时, 即使在底物苯甲腈过量的情况下也不易 进行 $\mathrm{C} \equiv \mathrm{N}$ 加成反应, 以 $\mathrm{C} \equiv \mathrm{C}$ 环三聚反应为主, 苯衍生 物和吡啶衍生物的产率比均大于 $3: 1$; 当选用 $\mathrm{Co}_{2}(\mathrm{CO})_{8}$ 为催化剂时, 只能催化炔烃环三聚反应, 产物 中也只有取代苯衍生物, 对炔-腈环加成反应没有催化 活性. 当使用 $\mathrm{ScCO}_{2}$ 代替甲苯作为炔-腈环加成反应溶 剂时, 苯衍生物的产率都有不同程度的提高. 这一结果 说明, 选择 $\mathrm{ScCO}_{2}$ 为反应溶剂, 不利于进行炔-腈环加 成反应，而更有利于进行炔一炔环三聚反应.

\section{2 炔烃环三聚反应机理}

在本文优化出的炔烃环三聚催化反应体系中, $\mathrm{Co}_{2}(\mathrm{CO})_{8}$ 为催化剂前体, 超临界二氧化碳起反应溶剂作 用, 反应不需要助溶剂和助催化剂. 根据文献报道 ${ }^{[15,17]}$ 及我们的实验结果, 推测的可能反应机理见 Scheme 1.

首先, $\mathrm{Co}_{2}(\mathrm{CO})_{8}$ 中的两个 $\mathrm{CO}$ 被一分子炔烃取代形 成配合物 $\mathbf{A}$. 配合物 $\mathbf{A}$ 再与另一分子炔烃侧基配位, 同 时失去一个 $\mathrm{CO}$, 形成中间体 $\mathbf{B}$, 然后发生分子内炔烃加 成配位反应，得到不对称和对称的环戊二烯钴配合物 $\mathbf{C}$ 或 $\mathbf{C}^{\prime}$. 配合物 $\mathbf{C}$ 或 $\mathbf{C}^{\prime}$ 再与第三个炔烃分子进行侧基配 位，同时失去一个 CO, 形成中间体 $\mathbf{D}$ 或 $\mathbf{D}^{\prime}$. 中间体 $\mathbf{D}$ 或 $\mathrm{D}^{\prime}$ 与侧基配位的炔烃发生分子内加成配位反应，得 到三种中间体 $\mathbf{E} 、 \mathbf{E}^{\prime}$ 或 $\mathbf{E}^{\prime}$. 最后，经过还原消除反应， 由中间体 $\mathbf{E}$ 和 $\mathbf{E}^{\prime}$ 得到 1,2,4-三取代苯衍生物 $\mathbf{a}$, 由中间 
表 3 炔一腈环加成反应 ${ }^{a}$

Table 3 Alkyne-nitrile cycloaddition reaction

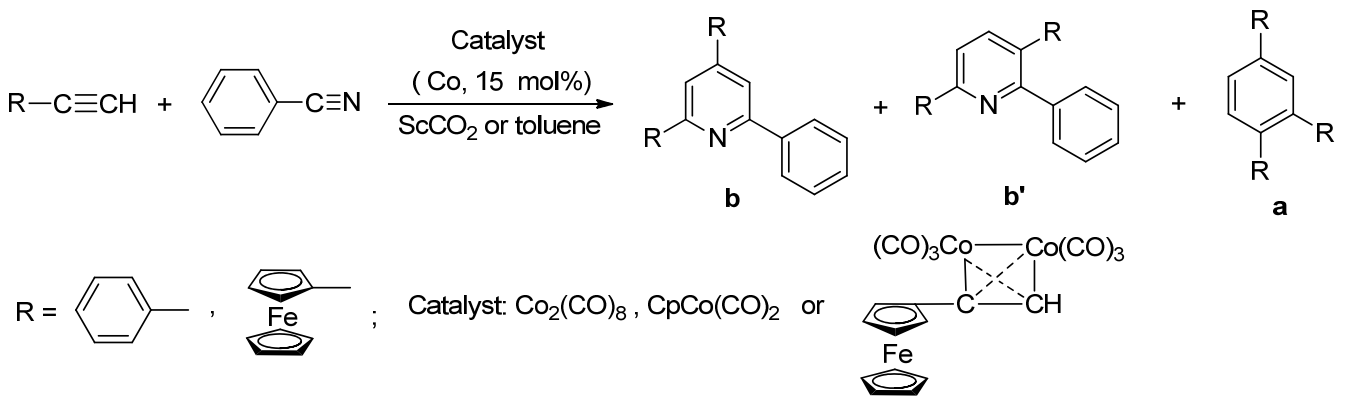

\begin{tabular}{|c|c|c|c|c|c|c|}
\hline \multirow{2}{*}{ Entry } & \multirow{2}{*}{ Alkyne $^{\mathrm{b}}$} & \multirow{2}{*}{ Catalyst } & \multirow{2}{*}{ Solvent } & \multicolumn{3}{|c|}{ Yield $^{c} \%$} \\
\hline & & & & b & $\mathbf{b}^{\prime}$ & $\mathbf{a}$ \\
\hline $1^{d}$ & 1 & $\mathrm{Co}_{2}(\mathrm{CO})_{8}$ & $\mathrm{ScCO}_{2}$ & - & - & 29 \\
\hline $2^{d}$ & 7 & $\mathrm{Co}_{2}(\mathrm{CO})_{8}$ & $\mathrm{ScCO}_{2}$ & - & - & 36 \\
\hline $3^{e}$ & 7 & $\mathrm{CpCo}(\mathrm{CO})_{2}$ & $\mathrm{ScCO}_{2}$ & 30 & 10 & 17 \\
\hline $4^{e}$ & 7 & $\mathrm{Co}_{2}(\mathrm{CO})_{6}\left(\mu_{2}-\eta^{2}-\mathrm{FcC} \equiv \mathrm{CH}\right)$ & $\mathrm{ScCO}_{2}$ & 8 & 2 & 51 \\
\hline $5^{f}$ & 7 & $\mathrm{Co}_{2}(\mathrm{CO})_{8}$ & Toluene & - & - & 55 \\
\hline $6^{f}$ & 7 & $\mathrm{CpCo}(\mathrm{CO})_{2}$ & Toluene & 51 & 22 & 10 \\
\hline $7^{f}$ & 7 & $\mathrm{Co}_{2}(\mathrm{CO})_{6}\left(\mu_{2}-\eta^{2}-\mathrm{FcC} \equiv \mathrm{CH}\right)$ & Toluene & 9 & 4 & 38 \\
\hline
\end{tabular}

${ }^{a}$ Reaction conditions: $\mathrm{FcC} \equiv \mathrm{CH}, \mathrm{PhC} \equiv \mathrm{CH}(0.48 \mathrm{mmol}) ; \mathrm{PhCN}(3.0 \mathrm{mmol})$; catalysts: $(\mathrm{Co}, 15 \mathrm{~mol} \%), \mathrm{Co}_{2}(\mathrm{CO})_{8}(0.035 \mathrm{mmol}), \mathrm{CpCo}(\mathrm{CO})_{2}(0.07 \mathrm{mmol})$, $\mathrm{Co}_{2}(\mathrm{CO})_{6}\left(\mu_{2}-\eta^{2}-\mathrm{FcC} \equiv \mathrm{CH}\right)(0.035 \mathrm{mmol})$; solvent: $\mathrm{ScCO}_{2}$ or toluene. ${ }^{b}$ Alkyne 1: $\mathrm{PhC} \equiv \mathrm{CH}$, alkyne 7 : $\mathrm{FcC} \equiv \mathrm{CH} .{ }^{c}$ Isolated yield. ${ }^{d} \mathrm{ScCO}{ }_{2}, 80{ }^{\circ} \mathrm{C}, 8 \mathrm{MPa}, 10 \mathrm{~h}$. ${ }^{e} \mathrm{ScCO}_{2}, 110{ }^{\circ} \mathrm{C}, 10 \mathrm{MPa}, 10 \mathrm{~h} .{ }^{f}$ Toluene, $110{ }^{\circ} \mathrm{C}, 5 \mathrm{~h}^{[16]}$.

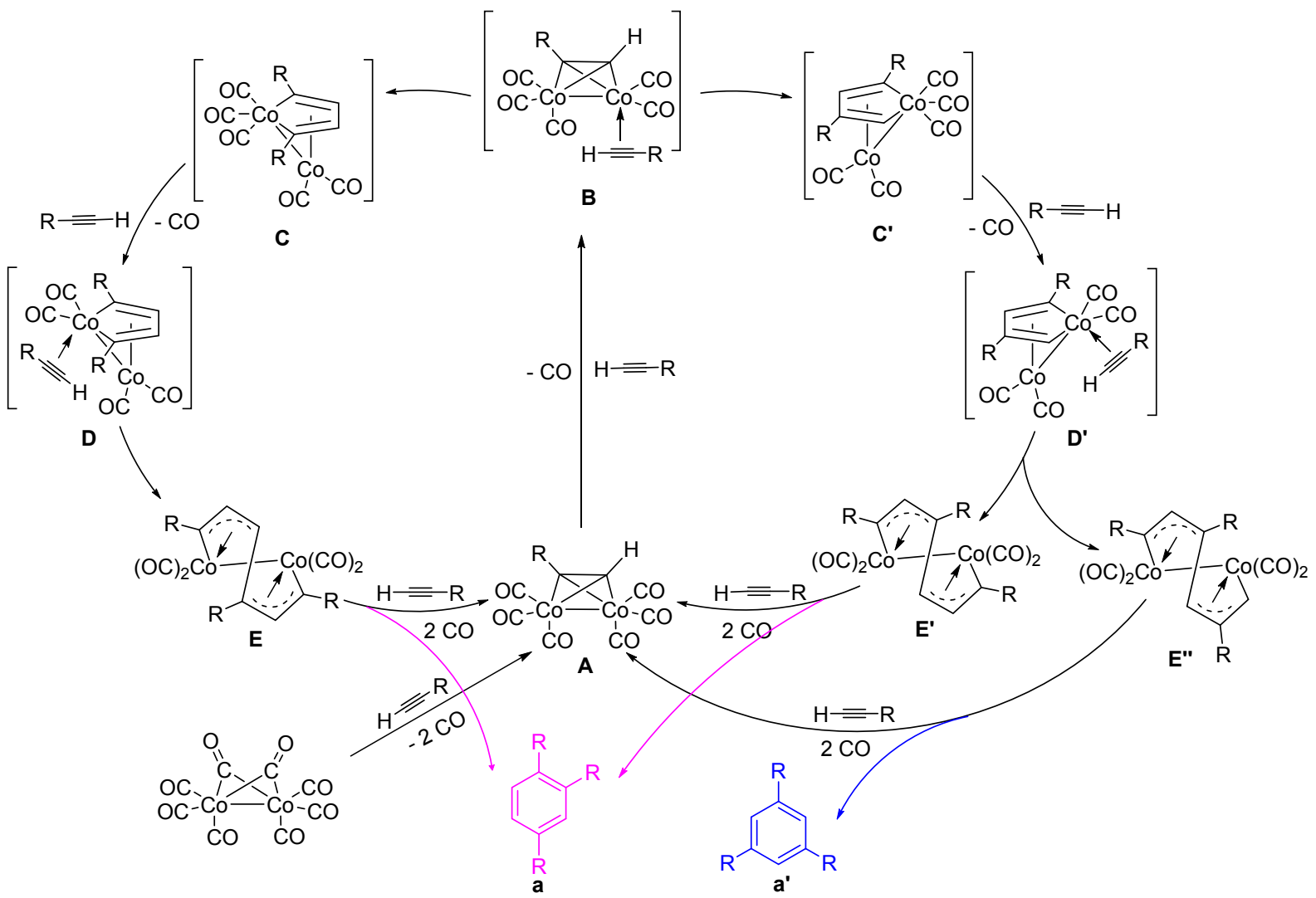

图式 $1 \mathrm{Co}_{2}(\mathrm{CO})_{8}$ 催化的端基炔烃环三聚反应机理推测

Scheme 1 Mechanistic assumption of the $\mathrm{Co}_{2}(\mathrm{CO})_{8}$ catalyzed cyclotrimerization of terminal alkynes 
体 $\mathbf{E}$ "得到了 1,3,5-三取代苯衍生物 $\mathbf{a}^{\mathbf{\prime}}$, 同时通过羰基钴 核与两分子 $\mathrm{CO}$ 和一分子炔烃配位, 使催化剂 $\mathbf{A}$ 再生, 从而开始新一轮的催化循环. 根据我们的推理, $\mathrm{Co}_{2}(\mathrm{CO})_{8}$ 为催化剂前体, 中间体 $\mathbf{A}$ 为催化剂的催化活性 中心.

从端基炔烃加成的角度来看, 形成配合物 $\mathbf{C}$ 比配合 物 $\mathbf{C}^{\prime}$ 占优势 ${ }^{[17 c, 18]}$, 推测存在三种中间体 $\mathbf{E} 、 \mathbf{E}^{\prime}$ 或 $\mathbf{E}^{\prime \prime}$, 其 中两种中间体 $\mathbf{E}$ 和 $\mathbf{E}^{\prime}$ 能生成 1,2,4-三取代苯产物, 只有 一种中间体 E"生成异构体 1,3,5-三取代苯, 1,2,4-三取代 苯产物选择几率较大, 这一推理与环三聚反应主要产物 是 1,2,4-三取代苯的实验结果一致.

\section{2 结论}

本文以 $\mathrm{Co}_{2}(\mathrm{CO})_{8}$ 为催化剂实现了在纯超临界二氧 化碳中的端基炔烃环三聚反应, 建立了一种合成 1,2,4三取代苯衍生物的新方法. 本文优化出的催化反应体系 具有催化剂廉价易得、反应溶剂环境友好、底物适应性 好、产物选择性高、容易分离、反应过程不使用有机溶 剂和各类助剂等优点. 反应物料溶解性和相变化的研究 结果表明, 超临界二氧化碳在反应过程中主要起到了反 应介质的作用, 反应物和催化剂在 $\mathrm{ScCO}_{2}$ 介质中的溶解 性和碰撞几率对反应产率和产物选择性影响很大, 不同 底物的产率高低与炔烃本身性质和溶解性有关. 底物拓 展实验结果表明, 在 $\mathrm{ScCO}_{2}$ 介质中, $\mathrm{CpCo}(\mathrm{CO})_{2}$ 和 $\mathrm{Co}_{2}(\mathrm{CO})_{6}\left(\mu_{2}-\eta^{2}-\mathrm{FcC} \equiv \mathrm{CH}\right)$ 催化剂是活化二茂铁基乙炔 与苯甲腈发生环加成反应的有效催化剂, 选用 $\mathrm{Co}_{2}(\mathrm{CO})_{8}$ 催化剂仅能得到取代苯衍生物, 不能得到取代吡啶衍生 物. $\mathrm{ScCO}_{2}$ 介质有利于促进炔烃环三聚反应而不利于进 行炔一腈环加成反应.

\section{3 实验部分}

\section{1 仪器与试剂}

河南省予华仪器有限公司 SGCX-4 显微熔点仪(温 度未校正); 美国 Nicolet FT-IR 红外光谱仪( $\mathrm{KBr}$ 压片); 日本 Shimadzu LCMS 2020 液相色谱一质谱联用仪; 美国 Thermo Fisher Polaris Q GC-MS 气相色谱-质谱联用仪; 美国 Agilent $500 \mathrm{MHz}$ DD2 核磁共振仪(TMS 作内标); 美国 Agilent 7200 Q-TOF GC-MS 高分辨质谱仪; 海安 县石油科研仪器有限公司提供高压反应釜 $(50 \mathrm{~mL}, 40$ $\mathrm{MPa})$ 和视窗反应釜(120 mL, $30 \mathrm{MPa})$.

实验操作均在标准的 Schlenk 技术(干燥的氩气)下 完成; 二氧化碳的纯度为 $99.99 \%$, 购于呼和浩特华远气 体有限公司; 所有端炔纯度均 $>97 \%$, 购于上海阿拉丁 试剂公司; $\mathrm{CpCo}(\mathrm{CO})_{2}$ (纯度 $>97 \%$ )购于阿法埃莎公司; $\mathrm{Co}_{2}(\mathrm{CO})_{8}($ 纯度 $>98 \%$ ) 购于中国科学院兰州化学物理研
究所; $\mathrm{Co}_{2}(\mathrm{CO})_{6}\left(\mu_{2}-\eta^{2}-\mathrm{FcC} \equiv \mathrm{CH}\right)$ 按照文献方法合成 ${ }^{[19]}$, 纯度 $>98 \%$. 所用有机溶剂均为分析纯，由天津市北联 精细化学厂提供, 使用前经过干燥或蒸馏处理. 柱层析 所用硅胶 (200 300 目) 和制备薄层层析用硅胶板 (GF254)均由青岛海洋化工有限公司提供.

\section{2 实验方法}

\subsection{1 炔烃环三聚反应实验方法}

向 $50 \mathrm{~mL}$ 带有磁力搅拌子的高压反应釜中加入 1.8 $\mathrm{mmol}$ 端炔 1 $\sim 8,0.14 \mathrm{mmol} \mathrm{Co}_{2}(\mathrm{CO})_{8}(47 \mathrm{mg}, \mathrm{Co}: 15$ $\mathrm{mol} \%$ ), 使用 Schlenk 技术以高纯氩气换气三次. 将反应 釜置于 $50{ }^{\circ} \mathrm{C}$ 的油浴中预热 $20 \mathrm{~min}$ 后, 充入纯 $\mathrm{CO}_{2}$ 气体, 使反应釜压力至 $8 \mathrm{MPa}$, 搅拌加热至 $80{ }^{\circ} \mathrm{C}$, 反应 $10 \mathrm{~h}$. 反应结束后, 冷却至室温, 缓慢卸去压力. 以 $\mathrm{CH}_{2} \mathrm{Cl}_{2}$ 溶 解反应釜中的样品, 减压除去溶剂后, 采用制备薄层色 谱法分离纯化产物 [洗脱剂为石油醚/ $\mathrm{CH}_{2} \mathrm{Cl}_{2}, V / V=2 / 1$ ], 得到相应的 1,2,4-三取代苯 $\mathbf{1 a} \sim 8 \mathbf{8}$.

1,2,4-三苯基苯(1a) ${ }^{[20]}$ : 白色固体, 产率 $62 \%$. m.p. 95 96 ${ }^{\circ} \mathrm{C} ;{ }^{1} \mathrm{H}$ NMR $\left(\mathrm{CDCl}_{3}, 500 \mathrm{MHz}\right) \delta: 7.58 \sim 7.55$ (m, 4H), $7.42(\mathrm{~d}, J=7.5 \mathrm{~Hz}, 1 \mathrm{H}), 7.36(\mathrm{t}, J=7.0 \mathrm{~Hz}, 2 \mathrm{H})$, 7.27 (t, $J=7.0 \mathrm{~Hz}, 1 \mathrm{H}), 7.13 \sim 7.08(\mathrm{~m}, 10 \mathrm{H}) ;{ }^{13} \mathrm{C} \mathrm{NMR}$ $\left(\mathrm{CDCl}_{3}, 125 \mathrm{MHz}\right) \delta: 140.43,140.06,139.94,139.53$, $139.31,138.50,130.06,128.86,128.83,128.38,127.79$, $126.89,126.86,126.39,126.09,125.55,125.48,125.08$; FT-IR (KBr) v: 3080, 3054, 3025, 1598, 1472, 1453, 757, $701 \mathrm{~cm}^{-1}$; MS (70 eV) $\mathrm{m} / \mathrm{z}(\%): 306.0\left(\mathrm{M}^{+}, 100\right), 289.1$ (27), 77.1 (4).

1,2,4-三(4-甲基苯基)苯(2a) ${ }^{[20]}$ : 白色固体, 产率 52\%. m.p. 90 91 ${ }^{\circ} \mathrm{C} ;{ }^{1} \mathrm{H}$ NMR $\left(\mathrm{CDCl}_{3}, 500 \mathrm{MHz}\right) \delta$ : $7.61 \sim 7.55$ (m, 4H), $7.46(\mathrm{~d}, J=7.5 \mathrm{~Hz}, 1 \mathrm{H}), 7.25(\mathrm{~s}, 2 \mathrm{H})$, $7.10 \sim 7.03(\mathrm{~m}, 8 \mathrm{H}), 2.40(\mathrm{~s}, 3 \mathrm{H}), 2.32 \sim 2.31(\mathrm{~m}, 6 \mathrm{H}) ;{ }^{13} \mathrm{C}$ $\mathrm{NMR}\left(\mathrm{CDCl}_{3}, 125 \mathrm{MHz}\right) \delta: 140.77,140.02,139.14$, 138.74, 138.34, 137.80, 137.12, 136.10, 136.00, 131.08, $129.72,129.68,129.51,129.25,128.66,128.63,126.93$, 125.69, 21.14, 21.12; FT-IR (KBr) v: 3025, 2920, 2853, $1517,1450,1379,810 \mathrm{~cm}^{-1}$; MS (70 eV) $\mathrm{m} / \mathrm{z}(\%): 348.0$ $\left(\mathrm{M}^{+}, 100\right), 333.1$ (35), 91.1 (13).

1,2,4-三(4-甲氧基苯基)苯 (3a $)^{[20]}$ : 白色固体, 产率 58\%. m.p. $102 \sim 104{ }^{\circ} \mathrm{C} ;{ }^{1} \mathrm{H}$ NMR $\left(\mathrm{CDCl}_{3}, 500 \mathrm{MHz}\right) \delta$ : $7.66(\mathrm{~d}, J=1.5 \mathrm{~Hz}, 1 \mathrm{H}), 7.65 \sim 7.60(\mathrm{~m}, 3 \mathrm{H}), 7.49(\mathrm{dd}, J=$ 1.5, 7.5 Hz, 1H), 7.19 (d, $J=2.0 \mathrm{~Hz}, 1 \mathrm{H}), 7.17$ (d, $J=2.5$ $\mathrm{Hz}, 1 \mathrm{H}), 7.16(\mathrm{~d}, J=2.5 \mathrm{~Hz}, 1 \mathrm{H}), 7.15(\mathrm{~d}, J=2.0 \mathrm{~Hz}, 1 \mathrm{H})$, $7.05 \sim 7.03(\mathrm{~m}, 2 \mathrm{H}), 6.86 \sim 6.82(\mathrm{~m}, 4 \mathrm{H}), 3.89(\mathrm{~s}, 3 \mathrm{H})$, $3.82(\mathrm{~s}, 6 \mathrm{H}) ;{ }^{13} \mathrm{C} \mathrm{NMR}\left(\mathrm{CDCl}_{3}, 125 \mathrm{MHz}\right) \delta: 159.17$, $158.28,158.20,140.34,139.54,138.41,134.10,133.69$, $133.15,130.96,130.88,130.83,128.85,128.04,125.32$, 
114.20, 113.39, 113.37, 55.27, 55.11, 55.10; FT-IR (KBr) $v:$ 3032, 3006, 2958, 2932, 2831, 1603, 1509, 1439, 821 $\mathrm{cm}^{-1}$; MS (70 eV) m/z (\%): $396.5\left(\mathrm{M}^{+}, 22\right), 395.4(\mathrm{M}-$ $\left.\mathrm{H}^{+}, 100\right), 91.3$ (7).

1,2,4-三(4-溴苯基) 苯(4a) ${ }^{[20]}$ : 白色固体, 产率 $40 \%$. m.p. $168 \sim 170{ }^{\circ} \mathrm{C} ;{ }^{1} \mathrm{H}$ NMR $\left(\mathrm{CDCl}_{3}, 500 \mathrm{MHz}\right) \delta: 7.62$ (dd, $J=2.0,8.0 \mathrm{~Hz}, 1 \mathrm{H}), 7.60 \sim 7.58(\mathrm{~m}, 2 \mathrm{H}), 7.56(\mathrm{~d}, J=$ $2.0 \mathrm{~Hz}, 1 \mathrm{H}), 7.53 \sim 7.50(\mathrm{~m}, 2 \mathrm{H}), 7.46(\mathrm{~d}, J=8.0 \mathrm{~Hz}, 1 \mathrm{H})$, $7.41 \sim 7.37(\mathrm{~m}, 4 \mathrm{H}), 7.07 \sim 7.00(\mathrm{~m}, 4 \mathrm{H}) ;{ }^{13} \mathrm{C} \mathrm{NMR}$ $\left(\mathrm{CDCl}_{3}, 125 \mathrm{MHz}\right) \delta$ : 139.83, 139.82, 139.68, 139.49, $139.11,138.53,132.03,131.40,131.38,131.35,131.34$, 131.14, 129.04, 128.65, 126.37, 121.99, 121.29, 121.21; FT-IR (KBr) v: 3025, 1588, 1465, $806 \mathrm{~cm}^{-1}$; MS (70 eV) $m / z(\%): 541.6\left(\mathrm{M}^{+}, 100\right), 543.5\left(\mathrm{M}^{+}+2 \mathrm{H}, 93\right), 381.8$ (90), 383.8 (80), 302.0 (60), 77.1 (13).

1,2,4-三(4-氟苯基) 苯 $(5 \mathbf{a})^{[21]}$ : 白色固体, 产率 $67 \%$. m.p. $146 \sim 148{ }^{\circ} \mathrm{C} ;{ }^{1} \mathrm{H} \mathrm{NMR}\left(\mathrm{CDCl}_{3}, 500 \mathrm{MHz}\right) \delta: 7.63 \sim$ $7.57(\mathrm{~m}, 4 \mathrm{H}), 7.46(\mathrm{~d}, J=8.0 \mathrm{~Hz}, 1 \mathrm{H}), 7.17 \sim 7.09(\mathrm{~m}$, $6 \mathrm{H}), 6.97 \sim 6.94(\mathrm{~m}, 4 \mathrm{H}) ;{ }^{13} \mathrm{C} \mathrm{NMR}\left(\mathrm{CDCl}_{3}, 125 \mathrm{MHz}\right) \delta$ : $163.61,162.85,162.81,161.64,160.89,160.85,140.02$, $139.63,138.50,137.13,137.10,136.77,136.74,136.48$, $136.45,131.38,131.34,131.32,131.28,131.07,129.17$, $128.70,128.63,126.16,115.85,115.68,115.13,115.10$, 114.96, 114.93; FT-IR (KBr) v: 3035, 1601, 1505, 1479, $815 \mathrm{~cm}^{-1}$; MS (70 eV) $\mathrm{m} / \mathrm{z}(\%): 360.2\left(\mathrm{M}^{+}, 100\right), 338.0$ (28), 250.9 (10).

1,2,4-三(3-吡啶基)苯(6a): 白色固体, 产率 44\%. m.p. $134 \sim 136{ }^{\circ} \mathrm{C} ;{ }^{1} \mathrm{H}$ NMR $\left(\mathrm{CDCl}_{3}, 500 \mathrm{MHz}\right) \delta: 8.95$ (s, $1 \mathrm{H}), 8.67(\mathrm{~s}, 1 \mathrm{H}), 8.52(\mathrm{~s}, 4 \mathrm{H}), 7.99 \sim 7.96(\mathrm{~m}, 1 \mathrm{H}), 7.75$ (dd, $J=2.0,8.0 \mathrm{~Hz}, 1 \mathrm{H}), 7.67(\mathrm{~d}, J=2.0 \mathrm{~Hz}, 1 \mathrm{H}), 7.59$ (d, $J=8.0 \mathrm{~Hz}, 1 \mathrm{H}), 7.48 \sim 7.42(\mathrm{~m}, 3 \mathrm{H}), 7.22 \sim 7.19(\mathrm{~m}, 2 \mathrm{H})$; ${ }^{13} \mathrm{C}$ NMR $\left(\mathrm{CDCl}_{3}, 125 \mathrm{MHz}\right) \delta: 150.26,150.23,149.01$, $148.43,148.32,148.21,138.30,138.06,137.13,137.11$, 136.97, 134.46, 131.59, 129.51, 127.27, 123.81, 123.08; FT-IR (KBr) v: 3032, 1565, 1465, $802 \mathrm{~cm}^{-1}$; HRMS (ESI) calcd for $\mathrm{C}_{21} \mathrm{H}_{16} \mathrm{~N}_{3}[\mathrm{M}+\mathrm{H}]^{+} 310.1339$, found 310.1331 .

1,2,4-三二茂铁基苯 $(7 a)^{[16]}$ : 橙色固体, 产率 $53 \%$. m.p. $255 \sim 257{ }^{\circ} \mathrm{C} ;{ }^{1} \mathrm{H}$ NMR $\left(\mathrm{CDCl}_{3}, 500 \mathrm{MHz}\right) \delta$ : 7.86 (s, $1 \mathrm{H}), 7.59$ (d, $J=7.5 \mathrm{~Hz}, 1 \mathrm{H}), 7.34$ (d, $J=7.5 \mathrm{~Hz}, 1 \mathrm{H}), 4.73$ (s, 2H), $4.38(\mathrm{~s}, 2 \mathrm{H}), 4.19 \sim 4.08(\mathrm{~m}, 23 \mathrm{H}) ;{ }^{13} \mathrm{C}$ NMR $\left(\mathrm{CDCl}_{3}, 125 \mathrm{MHz}\right) \delta: 136.83,136.14,134.98,131.21$, $128.87,123.59,88.20,87.97,85.44,70.89,70.63,69.71$, 69.63, 69.56, 68.91, 67.51, 67.40, 66.27; FT-IR (KBr) v: $3091,1609,1505,1434,1110,1025,817 \mathrm{~cm}^{-1}$; MS (ESI) $m / z: 630.0\left(\mathrm{M}^{+}\right)$.
1,2,4-三(1-戊基)苯 $(\mathbf{8 a})^{[22]}$ : 无色液体，产率 $57 \% .{ }^{1} \mathrm{H}$ NMR $\left(\mathrm{CDCl}_{3}, 500 \mathrm{MHz}\right) \delta: 7.04 \sim 6.81(\mathrm{~m}, 3 \mathrm{H}), 2.55(\mathrm{~s}$, $6 \mathrm{H}), 1.55$ (s, 6H), 1.35 (s, 12H), $0.90(\mathrm{~s}, 9 \mathrm{H}) ;{ }^{13} \mathrm{C} \mathrm{NMR}$ $\left(\mathrm{CDCl}_{3}, 125 \mathrm{MHz}\right) \delta: 140.32,129.21,128.93,125.68$, $35.58,32.75,32.31,32.04,31.67,31.27,31.10,22.61$, 22.58, 14.06; FT-IR (KBr) v: 3025, 2920, 2853, 1517, 1483, 1450, 1379, $809 \mathrm{~cm}^{-1}$; MS (70 eV) $\mathrm{m} / z(\%): 288.2$ $\left(\mathrm{M}^{+}, 49\right), 287.2\left(\mathrm{M}-\mathrm{H}^{+}, 100\right), 175.3$ (18), 119.6 (19), $91.3(7)$.

\subsection{2 炔一腈环加成反应实验方法}

在带有搅拌子的 $50 \mathrm{~mL}$ 高压反应釜中加入 0.48 $\mathrm{mmol}$ 二茂铁乙炔 $(100 \mathrm{mg}), 3.0 \mathrm{mmol}$ 苯甲腈 $(300 \mu \mathrm{L})$, $0.07 \mathrm{mmol} \mathrm{CpCo}(\mathrm{CO})_{2}(10 \mu \mathrm{L}, \mathrm{Co}: 15 \mathrm{~mol} \%)$ 或 0.035 $\mathrm{mmol} \mathrm{Co} 2(\mathrm{CO})_{6}\left(\mu_{2}-\eta^{2}-\mathrm{FcC} \equiv \mathrm{CH}\right)(18 \mathrm{mg}, \mathrm{Co}: 15 \mathrm{~mol} \%)$, 使用 Schlenk 技术以高纯氩气换气三次, 室温下充入 10 $\mathrm{MPa}$ 的纯 $\mathrm{CO}_{2}$ 气体, 加热至 $110{ }^{\circ} \mathrm{C}$, 反应 $10 \mathrm{~h}$. 反应结 束后, 冷却至室温, 卸去压力. 以 $\mathrm{CH}_{2} \mathrm{Cl}_{2}$ 溶解反应釜中 的样品, 减压除去溶剂. 反应混合物采用中性氧化铝 (200 300 目)进行柱层析分离纯化(石油醚/乙酸乙酯, $V / V=20 / 1)$, 得到化合物 $\mathbf{b}, \mathbf{b}^{\prime}, \mathbf{7} \mathbf{a}$.

2-苯基-4,6-二二茂铁基吡啶(b) ${ }^{[16]}$ : 橙色固体, m.p. 172 $174{ }^{\circ} \mathrm{C} ;{ }^{1} \mathrm{H}$ NMR $\left(\mathrm{CDCl}_{3}, 500 \mathrm{MHz}\right) \delta: 8.17 \sim 8.15$ $(\mathrm{m}, 2 \mathrm{H}), 7.59(\mathrm{~d}, J=1.5 \mathrm{~Hz}, 1 \mathrm{H}), 7.54 \sim 7.51(\mathrm{~m}, 2 \mathrm{H})$, $7.46 \sim 7.43(\mathrm{~m}, 1 \mathrm{H}), 7.41(\mathrm{~d}, J=1.5 \mathrm{~Hz}, 1 \mathrm{H}), 5.07(\mathrm{t}, J=$ $2.0 \mathrm{~Hz}, 2 \mathrm{H}), 4.83(\mathrm{t}, J=2.0 \mathrm{~Hz}, 2 \mathrm{H}), 4.46 \sim 4.42(\mathrm{~m}, 4 \mathrm{H})$, $4.10 \sim 4.09(\mathrm{~m}, 10 \mathrm{H}) ;{ }^{13} \mathrm{C}$ NMR $\left(\mathrm{CDCl}_{3}, 125 \mathrm{MHz}\right) \delta$ : $158.71,156.38,148.73,139.90,128.76,128.61,126.95$, $115.38,114.38,84.59,82.05,69.98,69.80,69.71,67.64$, 66.94; FT-IR (KBr) v: 3131, 1598, 1489, 1401, 1107, 1003, $820 \mathrm{~cm}^{-1}$; MS (ESI) $m / z: 524.1\left(\mathrm{M}^{+}+\mathrm{H}\right)$.

2-苯基-3,6-二二茂铁基吡啶 $\left(\mathbf{b}^{\prime}\right)^{[16]}$ : 橙色固体, m.p. $181 \sim 183{ }^{\circ} \mathrm{C} ;{ }^{1} \mathrm{H}$ NMR $\left(\mathrm{CDCl}_{3}, 500 \mathrm{MHz}\right) \delta: 8.02(\mathrm{~d}, J=$ $8.0 \mathrm{~Hz}, 1 \mathrm{H}), 7.43 \sim 7.41(\mathrm{~m}, 2 \mathrm{H}), 7.38(\mathrm{~d}, J=8.0 \mathrm{~Hz}, 1 \mathrm{H})$, $7.31 \sim 7.30(\mathrm{~m}, 3 \mathrm{H}), 4.98(\mathrm{~s}, 2 \mathrm{H}), 4.38$ (s, 2H), 4.17 (t, $J=$ $1.5 \mathrm{~Hz}, 2 \mathrm{H}), 4.12(\mathrm{t}, J=2.0 \mathrm{~Hz}, 2 \mathrm{H}), 4.10(\mathrm{~s}, 5 \mathrm{H}), 4.06$ (s, $5 \mathrm{H}) ;{ }^{13} \mathrm{C}$ NMR $\left(\mathrm{CDCl}_{3}, 125 \mathrm{MHz}\right) \delta: 156.17,156.03$, $140.99,138.80,129.96,129.38,127.57,127.54117 .92$, $85.54,84.41,70.04,69.78,69.67,69.58,68.15,67.57$; FT-IR (KBr) v: 3123, 1582, 1494, 1460, 1402, 1106, 1002, $821 \mathrm{~cm}^{-1}$; MS (ESI) $m / z: 524.1\left(\mathrm{M}^{+}+\mathrm{H}\right)$.

辅助材料(Supporting Information) 合成产物的 ${ }^{1} \mathrm{H}$ $\mathrm{NMR} 、{ }^{13} \mathrm{C}$ NMR 谱图、反应物料溶解性及相行为实验 图片. 这些材料可以免费从本刊网站(http://sioc-journal. $\mathrm{cn} /$ )上下载. 


\section{References}

[1] Mykhailiuk, P. K. Org. Biomol. Chem. 2019, 17, 2839.

[2] Chopade, P. R.; Louie, J. Adv. Synth. Catal. 2006, 348, 2307.

[3] (a) Agenet, N.; Buisine, O.; Slowinski, F.; Gandon, V.; Aubert, C.; Malacria, M. Cotrimerizations of Acetylenic Compounds, John Wiley \& Sons, Inc., New Jersey, 2007, pp. 1 302.

(b) Kotha, S.; Lahiri, K.; Sreevani, G. Synlett 2018, 29, 2342.

[4] Zhang, N.; Wang, Q.; Shi, W. Z. Introduction to Modern Chemical Industry, China Petrochemical Press Co. Ltd, Beijing, 2013, p. 284 (in Chinese).

(张娜, 王强, 时维振, 现代化工导论, 中国石化出版社, 北京, 2013, p. 284.)

[5] Xue, H.; Martyn, P. Chem. Soc. Rev. 2012, 41, 1428.

[6] Skouta, R. Green Chem. Lett. Rev. 2009, 2, 121.

[7] (a) Qi, Z. R.; Jiang, H. F. Prog. Chem. 2010, 22, 1274 (in Chinese). (戚朝荣, 江焕峰, 化学进展, 2010, 22, 1274.)

(b) Olmos, A.; Asensio, G.; Pérez, P. J. ACS Catal. 2016, 6, 4265.

(c) Li, J. H.; Jia, L. Q.; Jiang, H. F. Chin. J. Org. Chem. 2000, 20, 293 (in Chinese).

(李金恒, 贾兰齐, 江焕峰, 有机化学, 2000, 20, 293.)

(d) Liu, W. J.; Liang, Y.; Tang, S.; Li, J. H. Chin. J. Org. Chem. 2004, 24, 1553 (in Chinese)

(刘文杰, 梁云, 唐石, 李金恒, 有机化学, 2004, 24, 1553.)

[8] (a) Chatterjee, M.; Ishizaka, T.; Kawanami, H. Selective Hydrogenation in Supercritical Carbon Dioxide Using Metal Supported Heterogeneous Catalyst, American Chemical Society, Washington, DC, 2015, pp. 191 250 .

(b) Ichikawa, S.; Seki, T.; Ikariya, T. Adv. Synth. Catal. 2014, 356, 2643.

[9] (a) Wang, X.; Kawanami, H. Appl. Catal., A 2008, 349, 86. (b) Bourne, R. A.; Xue, H.; Martyn, P.; George, M. W. Angew. Chem., Int. Ed. 2010, 48, 5322.

[10] (a) Li, F. W.; Suo, Q. L.; Hong, H. L.; Zhu, N.; Wang, Y. Q.; Han, L. M. Tetrahedron Lett. 2014, 55, 3878.

(b) Li, F. W.; Suo, Q. L.; Hong, H. L.; Zhu, N.; Wang, Y. Q.; Han, L. M. Chin. J. Org. Chem. 2014, 34, 2172 (in Chinese). (李发旺, 索全伶, 洪海龙, 竺宁, 王亚琦, 韩利民, 有机化学, 2014, 34, 2172.)
[11] (a) Li, F. W.; Suo, Q. L.; Hong, H. L.; Zhu, N.; Wang, Y. Q.; Guo, L. L.; Han, L. M. J. Supercrit. Fluids 2014, 92, 70.

(b) Wang, Y. L.; Suo, Q. L.; Han, L. M.; Guo, L. L.; Wang, Y.; Li, F. W. Tetrahedron 2018, 74, 1918.

[12] (a) Cheng, J. S.; Jiang, H. F. Eur. J. Org. Chem. 2004, 643.

(b) Jiang, H. F. Curr. Org. Chem. 2005, 9, 289.

(c) Li, J. H. Acta Chim. Sinica 2004, 62, 341 (in Chinese).

(李金恒, 化学学报, 2004, 62, 341.)

(d) Montilla, F.; Avilés, T.; Casimiro, T.; Ricardo, A. A.; Ponte, M. N. D. J. Organomet. Chem. 2001, 632, 113.

[13] (a) Casimiro, T.; Montilla, F.; Garcia, S.; Avilés, T.; Raeissi, S.; Shariati, A.; Peters, C. J.; Ponte, M. N. D.; Aguiar-Ricardo, A. J. Supercrit. Fluids 2004, 31, 1.

(b) Kazemi, S.; Belandria, V.; Janssen, N.; Richon, D.; Peters, C. J.; Kroon, M. C. J. Supercrit. Fluids 2012, 72, 320.

(c) Long, J. J.; Cui, C. L.; Zhang, Y. Q.; Yuan, G. H. Dyes Pigm. 2015, 115,88 .

[14] Kaganovich, V. S.; Rybinskaya, M. I. J. Organomet. Chem. 1988, 344, 383.

[15] Baxter, R. J.; Knox, G. R.; Moir, J. H.; Pauson, P. L.; Spicer, M. D. Organometallics 1999, 18, 206.

[16] Wang, Y. Q.; Han, L. M.; Suo, Q. L.; Zhu, N.; Hao, J. M.; Xie, R. J. Polyhedron 2013, 54, 221.

[17] (a) Giuliana Gervasio, E. S. J. Organomet. Chem. 1993, 44, 203. (b) Cetini, G.; Gambino, O.; Rossetti, R.; Sappa, E. J. Organomet. Chem. 1967, 8, 149.

(c) Wakatsuki, Y.; Nomura, O.; Kitaura, K.; Morokuma, K.; Yamazaki, H. J. Am. Chem. Soc. 1983, 105, 1907.

(d) Peng, W.; Yi, Z.; Fan, Q. C.; Hao, F.; Xie, Y. M.; King, R. B.; Schaefer, I. H. F. Organometallics 2014, 33, 2352.

(e) Chen, Z.; Liu, J.; Evans, A. J.; Alberch, L.; Wei, A. Chem. Mater. 2012, 26, 941.

[18] Stockis, A.; Hoffmann, R. J. Am. Chem. Soc. 1980, 102, 2952.

[19] Pittman, C. U.; Smith, L. R. J. Organomet. Chem. 1975, 90, 203.

[20] Sugahara, T.; Guo, J. D.; Sasamori, T.; Nagase, S.; Tokitoh, N. Angew. Chem., Int. Ed. 2018, 57, 3499.

[21] Xu, L. M.; Yu, R. C.; Wang, Y. F.; Chen, J. H.; Yang, Z. J. Org. Chem. 2014, 44, 5744.

[22] Riache, N.; Dery, A.; Callens, E.; Poater, A.; Basset, J. M. Organometallics 2015, 34, 690 . 